\title{
Genetic Algorithm-Based Multi-Hop Routing to Improve the Lifetime of Wireless Sensor Networks
}

\author{
Adel Rajab \\ College of Computer Science and Information Systems \\ Najran University \\ Najran, Saudi Arabia \\ 77adel.rajab@gmail.com
}

\begin{abstract}
Wireless sensor networks are known for their monitoring and tracking application-specific operations. These operations diversely demand improvement in existing strategies and their parameters. One key parameter is energy usage during operations. Energy plays a vital role in each application, as the wireless sensor networks lack battery lifetime and energy resources. So, there is a need for an optimized and efficient routing method with regard to energy consumption in wireless sensor networks. For multi-hop routing, the genetic algorithm serves as a robust algorithm with diverse optimized routing plans to improve the lifespan for large-scale wireless sensor networks. In this paper, the genetic algorithm provides the optimized routes for data operations and improves the lifetime of wireless sensor networks by saving energy. The performance of the genetic algorithm is compared with the TEEN algorithm.
\end{abstract}

Keywords-wireless sensor networks; multi-hop; genetic algorithm; optimization; lifetime durability

\section{INTRODUCTION}

Wireless Sensor Networks (WSNs) are constructed by many self-organized and multifunctional mobile or non-mobile sensor nodes operating wirelessly in an area [1-4]. These sensor nodes possess inherited abilities to sense and transmit the gathered data towards the sink or base station. Therefore, the process of the data transmission towards the sink consumes more energy than the other processes such as data collection and data processing [5]. Since the sensor nodes have smallsized batteries, the overall performance of the sensor network depends on their lifetime. Additionally, the WSNs are prone to failure and can be deployed randomly in hard-to-reach areas. To counter this problem, multi-hop communication plays an essential role in improving network lifetime [6-8]. In the multihop communication paradigm, the source node transmits the data through the intermediate sensor nodes. These intermediate sensor nodes relay the data towards the sink as depicted in Figure 1. Multi-hops minimize the energy consumption by consuming a tiny amount of energy to relay the data towards the neighbor and the nearest sensor nodes $[9,10]$, which are hard to find in the WSNs if the optimal routing path is not determined. The optimal path towards the nearest sensor nodes is the key to save the extra consumption of the energy of the node.
The Genetic Algorithm (GA) is used to save energy and determine all the possible paths in the WSNs. The multi-hop communication paradigm with a focus on reduction of energy usage and optimal paths towards the nearest relay nodes is based on the distance among them. So, the distance among the sensor nodes from the source node towards the sink, all relay sensor nodes, and a possible number of multiple hops are determined by the GA in order to select the most optimal path for the data transmission. The results with the utilization of the GA prove that the multi-hop communication paradigm improves the WSNs lifetime [11-15].

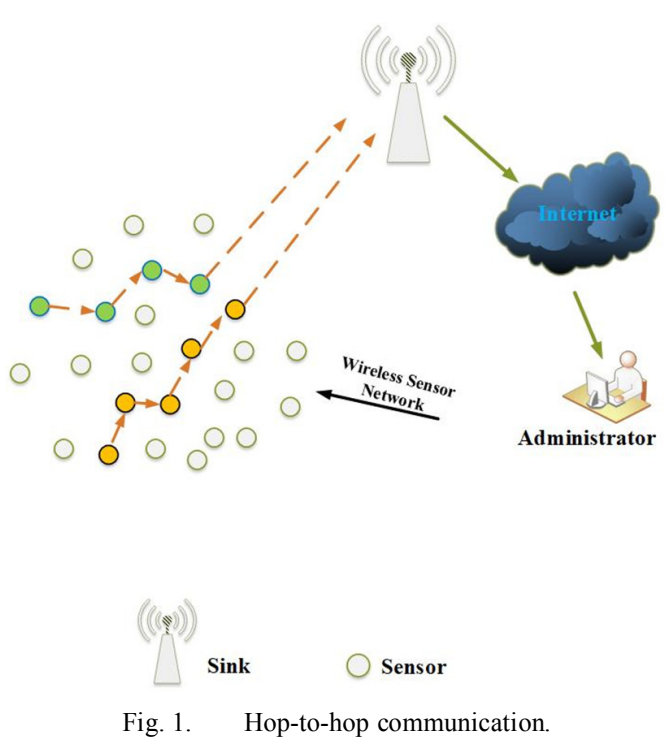

II. RELATED WORK

There is abundant research literature in the field of WSNs with regard to routing mechanisms and the improvement of energy utilization. Multipath routing schemes have been proposed to replace the single path routing paradigms. Replacing the single path or direct communication paradigms provides better performance of the network [8-11]. Multipath routing provides the benefit to transmit the packets by more than two paths, thus the packet loss rate is minimized $[11,12]$. On the other hand, the multipath technique reduces the chance 
of malicious attacks and any kind of packet tempering [13]. Multipath routing schemes for WSNs based on the next hop have been proposed in [14-16]. The sensor nodes are deployed in a hierarchical form and the algorithms are set to send the message according to the pre-determined loop of the hierarchical form. The authors claimed that this resulted in better network performance and more saved energy. Additionally, the routing schemes involving multiple paths reduced fault tolerance including error detection and error recovery. Authors in [17] presents a solution to the problem of the unbalanced energy expenditure in WSNs for multipath routing. The multiple-path routing scheme has been carried out in 3-D space and regional co-evolution. A protocol was designed to divide the one-hop path of the neighbor sensor node into many subspaces by partitions of the region. Then the source node selects the optimal node and path to transmit the data. The co-evolution algorithm helps to find out the next hop. A multipath cost function model for the statistical estimation of the overall network, namely the total energy of the nodes and the energy used in relaying the data was proposed in [18-20]. Multipath routing for the directional diffusion routing protocol from the source to the destination node with a certain probability of selecting one path among all possible paths was proposed in [19, 21-24]. Authors in [25, 27] advocated loadbalancing energy schemes for WSNs and energy harvesting for the overall network. They utilized ACO (Ant Colony Optimization) for multiple path finding and claimed satisfying results. The study used an electromagnetic antenna-based approach for energy harvesting to gain high performance of IoT and WSNs. Numerous studies have been carried out on minimum energy consumption. The efficient usage of energy has been claimed as an important part to maximize the WSNs lifetime through optimization [28-30]. The optimization also reduces the complexity of the overall network and provides many possible options for routing the data from source to destination.

\section{GENETIC ALGORITHM}

GAs are meta-heuristic algorithms. The GA mimics the natural selection process [21] and has been used in various computer and AI applications. Due to its robustness, many research scholars adopted GA for solving optimization problems. Optimization finds the possible solutions to the problems and potential methods to tackle complications. As the GA mimics the natural selection process it follows the selection of healthy chromosomes. These chromosomes are selected by the fitness function, and are subjected to crossover and mutation [22-24]. The working mechanism of GA in this research is utilized with some modifications. All the operators of GA are used for selecting the best individuals from the population. The population comprises of all sensor nodes and sinks deployed in the area. After the selection of the best population, the GA finds fit individuals by applying the fitness function as shown in Figure 2. Each individual passes on the basis of their energy. Then, other operators, namely crossover and mutation, are applied to select the best chromosome as shown in Figure 3(a)-(b). For this research, the mutation probability is set to $0.8[2,24]$. The process of mutation is depicted in Figure 3(c). After the mutation operator, the best individual and the new fit individual are re-evaluated on the basis of their energy and only the fit individual can be obtained.

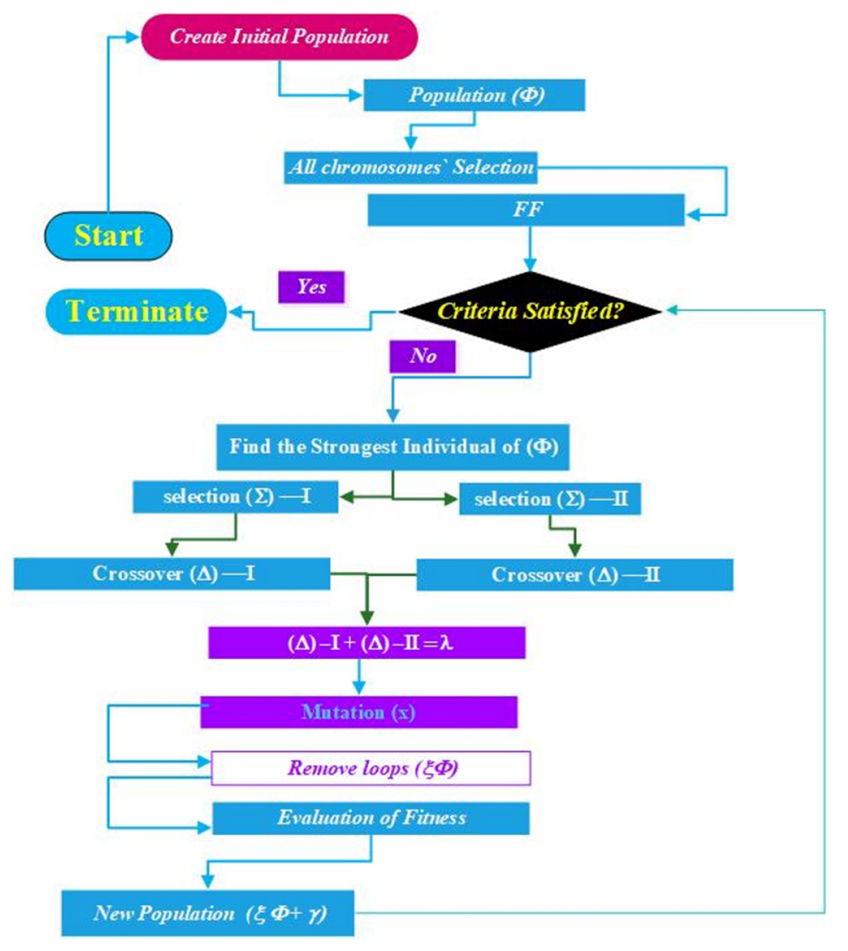

Fig. 2. Operator utilization of GA.

(a)

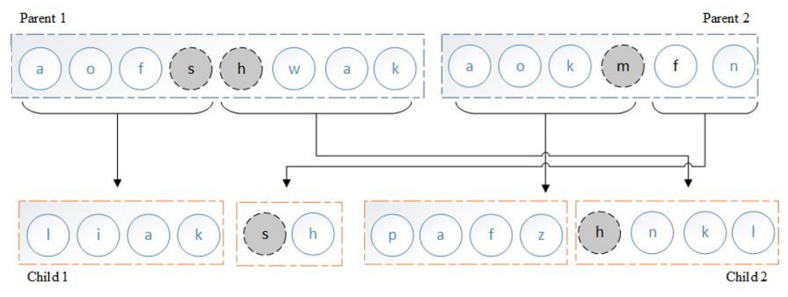

(c)

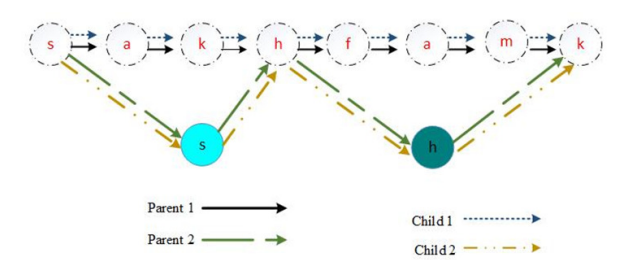

Fig. 3. (a), (b) Crossover and (c) mutation.

\section{PERFORMANCE AND SIMULATION}

The experimental setup includes a dual-core Intel processor with a CPU of $3.5 \mathrm{GHz}$ and $32 \mathrm{~GB}$ RAM. Simulations have been carried out using MATLAB R2020b in Windows 10 . The sensor nodes are simulated as non-mobile but randomly deployed in a $100 \times 100 \mathrm{~m}^{2}$ area with 4 sinks deployed at the corners. The multi-hop communication paradigm where the source node transmits the data through the relay nodes towards the nearest sink is shown in Figure 4. All possible routing paths towards all sinks are determined by the GA. When a node gathers and transmits the data by the optimal path towards the 
nearest sink, it follows the already optimized route from multiple possible routes determined by the GA. The multiple hop distance is evaluated and determined by the GA. All sinks broadcast their locations to all sensor nodes and the nodes only transmit the data towards the nearest sink as illustrated in Figure 4. The simulation results comprise 40 and 50 sensor nodes deployed in a static manner along with static sinks [26]. The results of alive nodes, dead nodes, and overall packet transmission are compared with the standard TEEN algorithm's and they fairly outperform it in terms of energy expenditure and WSNs lifetime maximization.

\section{A. Simulation Paramaters}

The studied WSN parameters are given in Table I. Each sink is responsible to collect the data from the nodes efficiently through optimized routes. Route optimization and determination are done with the help of GA. The evaluation of the route is done in two manners, namely the direct distance evaluation and the multiple hop distance evaluation. The evaluation of the distance is based upon the energy consumption. It is observed that the nearest nodes have the least energy consumption in comparison with the more remote nodes. During the simulations, the most remote nodes died in a few rounds because of the long routes and direct distances.

TABLE I. SIMULATION PARAMETERS

\begin{tabular}{|l|c|}
\hline \multicolumn{1}{|c|}{ Parameters } & Value \\
\hline Size of the network & $100 \times 100 \mathrm{~m}^{2}$ \\
\hline Number of nodes & 40 and 50 \\
\hline Initial energy & $0.9 \mathrm{Joules}$ \\
\hline Rounds & 4000 \\
\hline Dissipation of energy & $50 \mathrm{~nJ} / \mathrm{bit}$ \\
\hline Increase of energy $d_{i}<d_{j}$ & $10 \mathrm{pJ} / \mathrm{bit} / \mathrm{m}$ \\
\hline Increase of energy $d_{i}>d_{j}$ & $0.0015 \mathrm{pJ} / \mathrm{bit} / \mathrm{m}$ \\
\hline
\end{tabular}

\section{B. Simulation Algorithm}

The proposed algorithm provides the routing mechanism among all sensor nodes and all sinks. It initially provides all the sensor nodes with a number of trial rounds. These trials are based on the energy levels of every sensor node and the distance among the sensor nodes and respective sinks. Therefore, the distance and their selection of routes strictly depend upon their physical location, where they are deployed, and the nearest sink.

\section{Pseudo code for the optimization.}

$\begin{array}{ll}\text { Input: All Nodes: } & \text { Sensor }_{\text {Total }} \\ \text { Total trials: } & \emptyset \\ \text { Battery life of each node: } & \emptyset_{\text {first }} \\ \text { Network area: } & 100 \times 100 \mathrm{~m}^{2} \\ \text { Selected route and every route } i: & \partial_{i} \\ \text { Path optimization: } & \text { Poptim } \\ \text { for } i \text { from 0 until } \partial \text { do } & \\ \text { for } j \text { from 1until } \emptyset \text { do } & \\ \text { Sensor }_{\text {Total }} & \text { Produce random network } \\ \text { Sink }_{\text {Loc }} & \text { Sink locations are broadcasted. } \\ \text { Initial }_{\text {Route }}=0 & \text { for every new network } \\ \aleph_{\text {Routes }} & \text { Route determining from/to sink(s) }\end{array}$

end if

$\pi_{\text {Optimized Routes }} \quad$ Obtaining optimized routes

$\beta_{\text {Most optimal Route }}$ reservation of optimal route

do while $\partial \emptyset_{\text {first }}>0$ operation of nodes until battery dies

$\beta_{\text {Most optimal Route }}$ save the optimal route

$\beta_{\text {Most Optimal Route }} \quad$ copy it for every iteration.

end and return with $\beta_{\text {Most optimal Route }}$

\section{SimUlation RESUlts}

The WSN is constructed with 40 and 50 sensor nodes and 4 sink stations located at the corners of the network area as illustrated in Figures 5-6. The evaluation and simulation results of the randomly and static deployed sensor nodes and sinks show that the route distance is covered by multiple hop routing. That enhances the sensor node's battery lifetime and improves the network's lifetime. In other words, the network lifetime has, extensively, been increased by the use of the optimized multiple hop routes as depicted in Figure 7. Therefore, it has been experimentally proved that the network lifetime can be improved through optimum data transmission. Although there are a number of routes in multiple hop routing mechanism the source sensor node only opts to transmit the data through the optimal one.

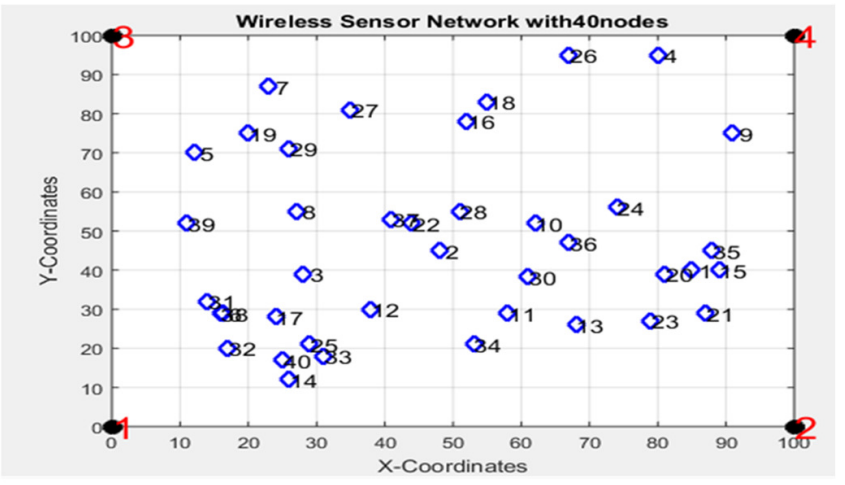

Fig. 4. Deployment of 40 sensor nodes and sinks.

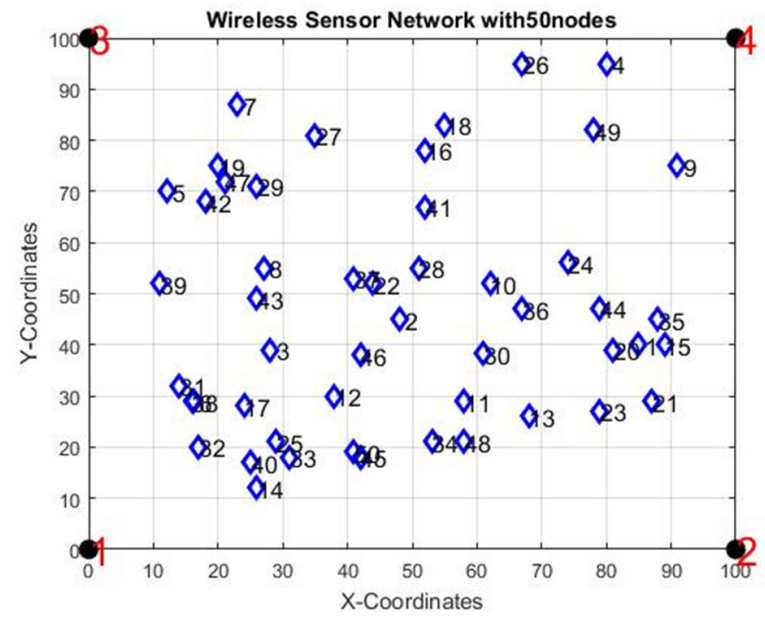

Fig. 5. Deployment of 50 sensor nodes and sinks. 


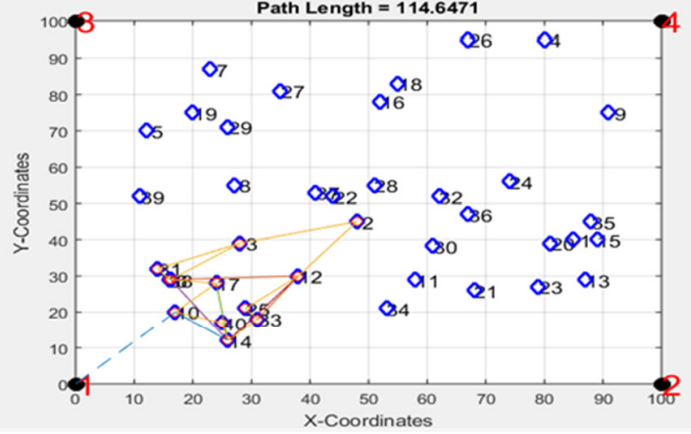

(a)

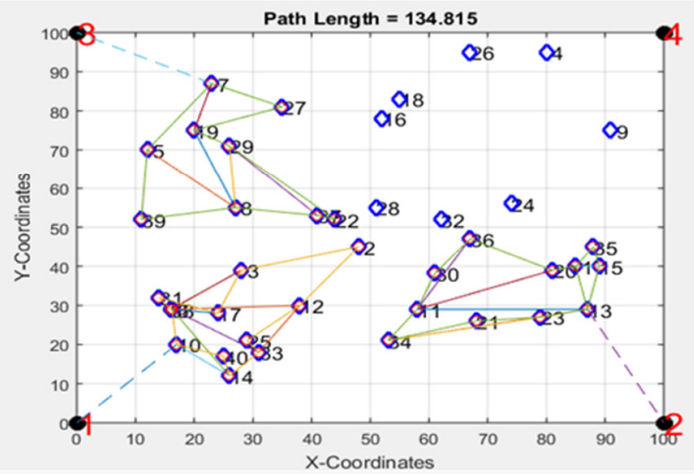

(c)

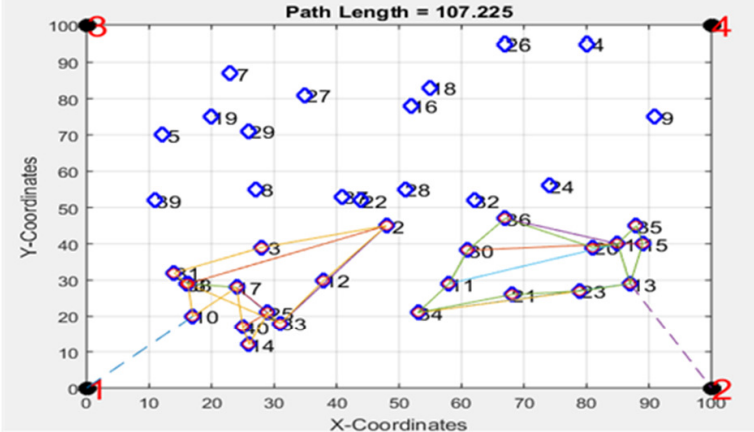

(b)

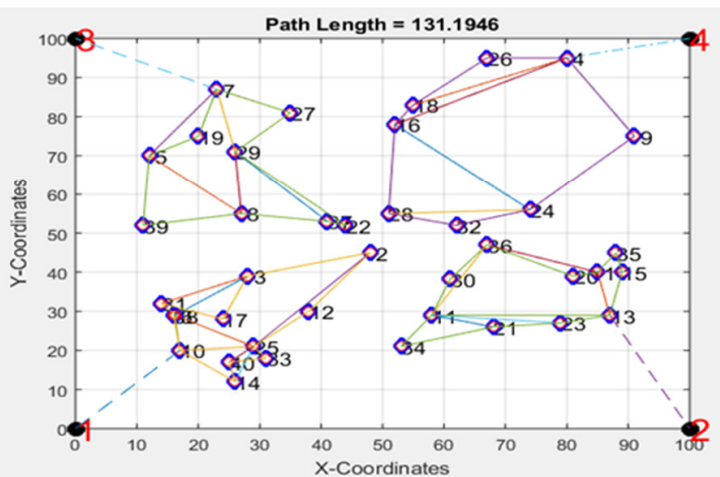

(d)

Fig. 6. Data transmission to (a) Sink 1 (b) Sink 2, (c) Sink 3, and (d) Sink 4.

The localization of 40 sensor and multi-hop relay sensor nodes is shown in Figure 7. Multiple paths are determined by the GA among all the sensor nodes and sinks. The sensor nodes in the nearest sensor node towards the sink receive the data and perform the execution. The source sends the data towards the nearest sink with the help of relay sensor nodes. The optimal distance towards Sink 1 and Sink 2 is $114.647 \mathrm{~ms}$ and $107.225 \mathrm{~m}$ respectively. Sink 3 with $134.815 \mathrm{~m}$ and Sink 4 with $131.194 \mathrm{~m}$ have optimal routes towards the farthest node.

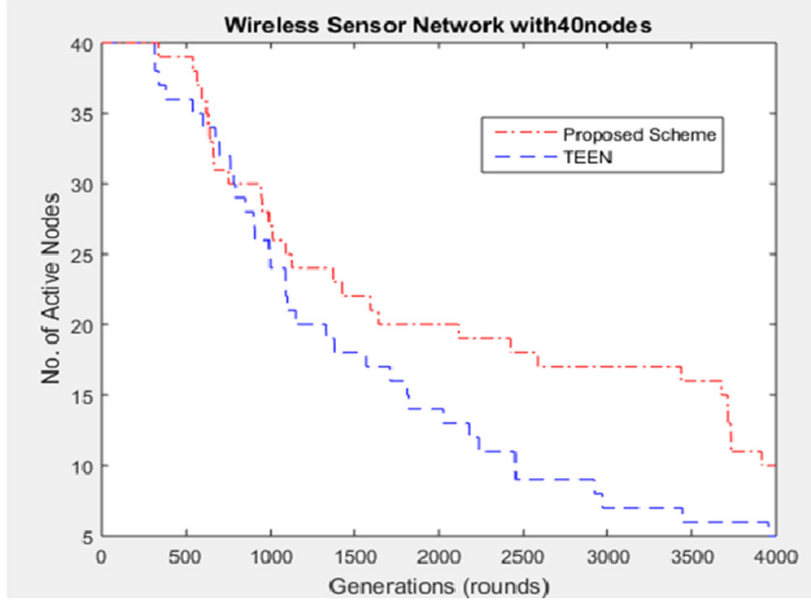

Fig. 7. Number of rounds vs. number of alive nodes for the proposed scheme and the TEEN algorithm.

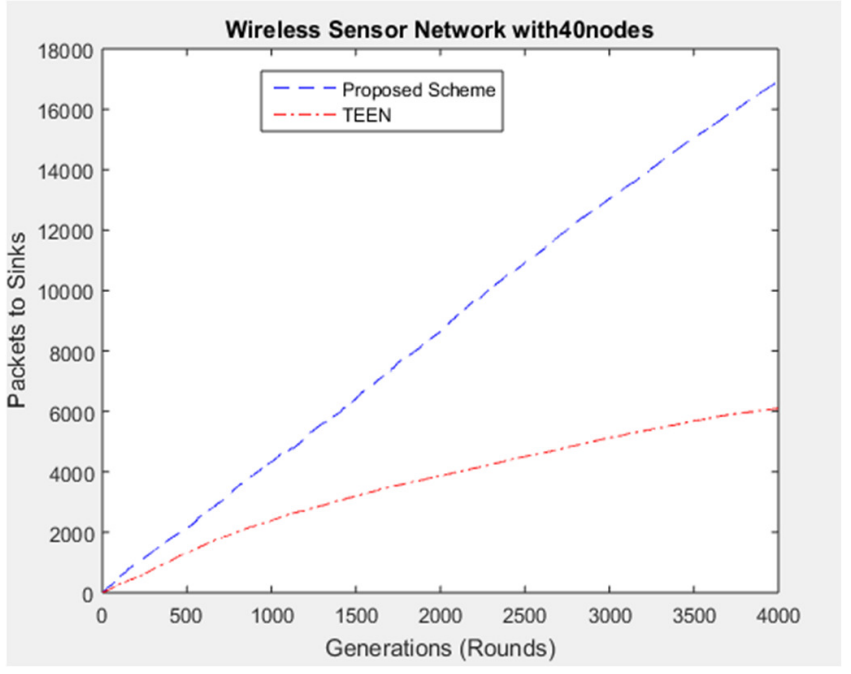

Fig. 8. Comparison of packets to sinks.

The result comparison of this research with the standard TEEN algorithm [26] shows that the proposed scheme performs significantly better. The results show that the number of rounds vs. the number of alive nodes of the proposed scheme is bigger than the respective number of the TEEN standard algorithm as shown in Figure 8. The data transmission towards the sinks and the comparison of the results with the standard TEEN algorithm are shown in Figure 9. The packets with the proposed scheme at the rounds 1500, 2000, 2500, 3000 , and 3700 have been received successfully at the end of 
the sink. Also, the proposed scheme maximized the WSN's life durability and improved network performance. Additionally, the comparison results show that the standard TEEN algorithm has significantly more dead nodes as the packet transmission increases, while the proposed algorithm performs better and increases network durability [26].

\section{DISCUSSION}

The proposed scheme improves the lifetime of the WSNs. The GA determines all possible routes for data transmission and optimizes each route for efficient data delivery with minimum energy cost. The GA determines an optimized route for each sensor node. The simulation results are more compromising than the standard TEEN [26] algorithm's. The proposed work performed very well on 500, 1000, 2000, and 3700 rounds and enhanced network lifetime.

\section{CONCLUSIONS AND FUTURE WORK}

In this study, a multi-hop routing scheme for wireless sensor networks, based on the energy optimization by GA for network lifetime maximization, has been proposed and examined. The GA provides the optimized routes for data operations and improves the lifetime of WSNs by saving energy. The simulation results demonstrate the robust performance of the GA resulting in improved network lifetime. Also, the proposed algorithm achieves a significant performance gain over the standard TEEN protocol. Networks with a significant number of sensor nodes were simulated as non-mobile WSNs. Comparatively, the GA lost less nodes in 2000, 3000, and 4000 rounds, than the standard TEEN algorithm. In simulation results, the GA performed fairly better than the standard TEEN algorithm. Regarding future work, the current study can be extended to mobile and hybrid optimization schemes.

\section{REFERENCES}

[1] J. Zhang, "Wireless Sensor Networks: Lecture 1," Norrköping, Sweden, Jan. 17, 2014.

[2] C. Li, J. Hailing, M. Yong, L. Tianpu, L. Wei, and and Z. Ze, "Overview of Wireless Sensor Networks," Journal of Computer Research and Development, vol. 42, no. 1, pp. 163-174, Jan. 2005.

[3] F. Samad, Q. Abu Ahmed, A. Shaikh, and A. Aziz, "JAM: Mitigating Jellyfish Attacks in Wireless Ad Hoc Networks," in Emerging Trends and Applications in Information Communication Technologies, 2012, pp. 432-444, https://doi.org/10.1007/978-3-642-28962-0_41.

[4] A. Shaikh, N. Anjum, F. Samad, and A. Shah, "Building Wireless Sensor Networks Application Using Sun SPOTs," in Emerging Trends and Applications in Information Communication Technologies, 2012, pp. 466-477, https://doi.org/10.1007/978-3-642-28962-0_44.

[5] M. A. Elmagzoub, A. Shaikh, A. Alghamdi, and K. Rajab, "A Review on MIMO Wireless Signals over Fibre for Next Generation Fibre Wireless (FiWi) Broadband Networks," Electronics, vol. 9, no. 12, Dec. 2020, Art. no. 2014, https://doi.org/10.3390/electronics9122014.

[6] S. Solangi, D. Hakro, A. Lashari, K.-U.-R. Khoumbati, Z. Bhutto, and M. Hameed, "Genetic Algorithm Applications in Wireless Sensor Networks (WSN): A Review," International Journal of Management Sciences and Business Research, vol. 6, no. 4, pp. 152-166, Apr. 2017.

[7] S. P. Singh and S. C. Sharma, "A Novel Energy Efficient Clustering Algorithm for Wireless Sensor Networks," Engineering, Technology \& Applied Science Research, vol. 7, no. 4, pp. 1775-1780, Aug. 2017, https://doi.org/10.48084/etasr.1277.

[8] G. Huang, W. Tao, P. Liu, and S. Liu, "Multipath ring Routing in Wireless Sensor Networks," presented at the 2nd International
Symposium on Computer, Communication, Control and Automation (ISCCCA 2013), Feb. 2013, pp. 768-771, https://doi.org/10.2991/ isccca.2013.193.

[9] I. F. Akyildiz and M. C. Vuran, Wireless Sensor Networks, 1st ed. Chichester, West Sussex, UK; Hoboken, NJ, USA: Wiley, 2010.

[10] P. Chanak and I. Banerjee, "Energy efficient fault-tolerant multipath routing scheme for wireless sensor networks," The Journal of China Universities of Posts and Telecommunications, vol. 20, no. 6, pp. 42-61, Dec. 2013, https://doi.org/10.1016/S1005-8885(13)60107-7.

[11] S. Wang, "Multipath Routing Based on Genetic Algorithm in Wireless Sensor Networks," Mathematical Problems in Engineering, vol. 2021, Jun. 2021, Art. no. e4815711, https://doi.org/10.1155/2021/4815711.

[12] N. A. Alrajeh, M. S. Alabed, and M. S. Elwahiby, "Secure Ant-Based Routing Protocol for Wireless Sensor Network," International Journal of Distributed Sensor Networks, vol. 9, no. 6, Jun. 2013, Art. no. 326295, https://doi.org/10.1155/2013/326295.

[13] L. J. and J. Huo, "Uneven Clustering Routing Algorithm Based on Optimal Clustering for Wireless Sensor Networks," Journal of Communications, vol. 11, no. 2, pp. 132-142, Feb. 2016, https://doi.org/ $10.12720 / \mathrm{jcm} .11 .2 .132-142$.

[14] S. Md Zin, N. Badrul Anuar, M. L. Mat Kiah, and I. Ahmedy, "Survey of secure multipath routing protocols for WSNs," Journal of Network and Computer Applications, vol. 55, pp. 123-153, Sep. 2015, https://doi.org/10.1016/j.jnca.2015.04.018.

[15] P. J. Morrissey, K. S. Vunnava, J. N. Potts, J. W. Ehm, and R. P. Singh, "Multi-path routing control for an encrypted tunnel," US9755953B1, Sep. 05, 2017.

[16] T. Murakami, E. Kohno, and Y. Kakuda, "Radio Overlapping Reduced Multipath Routing Method by Utilizing Control Packet Overhearing to Counter Eavesdropping on Data Packets for Ad Hoc Networks," in 2015 Third International Symposium on Computing and Networking (CANDAR), Sapporo, Japan, Dec. 2015, pp. 167-173, https://doi.org/ 10.1109/CANDAR.2015.56.

[17] W. Bai, H. Wang, X. Shen, R. Zhao, and Y. Zhang, "Minimum Delay Multipath Routing Based on TDMA for Underwater Acoustic Sensor Network," International Journal of Distributed Sensor Networks, vol. 12, no. 2, Feb. 2016, Art. no. 1394340, https://doi.org/10.1155/ $2016 / 1394340$.

[18] K. B. Wand and L. L. Cheng, "Wireless sensor network redundancy sensor node state schedule method," Aplication Research of Computers, vol. 35, no. 4, pp. 1227-1230, 2018.

[19] M. Zhou, Y. Wang, Y. Liu, and Z. Tian, "An Information-Theoretic View of WLAN Localization Error Bound in GPS-Denied Environment," IEEE Transactions on Vehicular Technology, vol. 68, no. 4, pp. 4089-4093, Apr. 2019, https://doi.org/10.1109/TVT.2019. 2896482.

[20] Z. Y. Zhang, Y. J. Liu, and X. D. Wang, "Multi-path routing in multifactor wireless sensor monitoring network," Computer Engineering \& Science, vol. 36, no. 6, pp. 1064-1071, Jun. 2014.

[21] W. Ma, F. Yan, X. Zuo, L. Ren, W. Xia, and L. Shen, "Coverage hole detection algorithm without location information in wireless sensor networks," in 2017 rd IEEE International Conference on Computer and Communications (ICCC), Chengdu, China, Dec. 2017, pp. 357-361, https://doi.org/10.1109/CompComm.2017.8322571.

[22] M. Zhou, Y. Wang, Z. Tian, Y. Lian, Y. Wang, and B. Wang, "Calibrated Data Simplification for Energy-Efficient Location Sensing in Internet of Things," IEEE Internet of Things Journal, vol. 6, no. 4, pp. 6125-6133, Aug. 2019, https://doi.org/10.1109/JIOT.2018.2869671.

[23] M. Zhou, X. Li, Y. Wang, S. Li, Y. Ding, and W. Nie, "6G MultisourceInformation-Fusion-Based Indoor Positioning via Gaussian Kernel Density Estimation," IEEE Internet of Things Journal, vol. 8, no. 20, pp. 15117-15125, Oct. 2021, https://doi.org/10.1109/JIOT.2020.3031639.

[24] W. R. Heinzelman, A. Chandrakasan, and H. Balakrishnan, "Energyefficient communication protocol for wireless microsensor networks," in Proceedings of the 33rd Annual Hawaii International Conference on System Sciences, Maui, HI, USA, Jan. 2000, https://doi.org/ 10.1109/HICSS.2000.926982. 
[25] A. Nayyar and R. Singh, "IEEMARP- a novel energy efficient multipath routing protocol based on ant Colony optimization (ACO) for dynamic sensor networks," Multimedia Tools and Applications, vol. 79, no. 47, pp. 35221-35252, Dec. 2020, https://doi.org/10.1007/s1 1042-019-7627-

[26] A. Manjeshwar and D. P. Agrawal, "TEEN: a routing protocol for enhanced efficiency in wireless sensor networks," in Proceedings 15th International Parallel and Distributed Processing Symposium. IPDPS 2001, San Francisco, CA, USA, Apr. 2001, pp. 2009-2015, https://doi.org/10.1109/IPDPS.2001.925197.

[27] I. Mujahidin and A. Kitagawa, "The Novel CPW $2.4 \mathrm{GHz}$ Antenna with Parallel Hybrid Electromagnetic Solar for IoT Energy Harvesting and Wireless Sensors," International Journal of Advanced Computer Science and Applications, vol. 12, no. 8, pp. 393-400, 2021, https://doi.org/ 10.14569/IJACSA.2021.0120845.

[28] J. O. Obira and R. Sinde, "Development of a Sensor-Based Heartbeat and Body Temperature Monitoring System for Remote Chronic Patients," Engineering, Technology \& Applied Science Research, vol. 11, no. 4, pp. 7375-7380, Aug. 2021, https://doi.org/10.48084/etasr. 4216.

[29] M. K. Sastry, A. A. K. Mohammad, and A. M. Abdul, "Optimized Energy-efficient Load Balance Routing Protocol for Wireless Mesh Networks," International Journal of Advanced Computer Science and Applications (IJACSA), vol. 12, no. 8, 2021, https://doi.org/10.14569/ IJACSA.2021.0120871.

[30] M. B. Apsara, P. Dayananda, and C. N. Sowmyarani, "A Review on Secure Group Key Management Schemes for Data Gathering in Wireless Sensor Networks," Engineering, Technology \& Applied Science Research, vol. 10, no. 1, pp. 5108-5112, Feb. 2020, https://doi.org/10.48084/etasr.3213. 